\title{
Thermal stress at wafer contact points in rapid thermal processing investigated by repeated spike treatment before oxidation
}

\author{
Chao-Chi Hong, Chang-Yun Chang, Chaung-Yuan Lee, and Jenn-Gwo Hwua) \\ Department of Electrical Engineering/Graduate Institute of Electronics Engineering, Room 446, \\ National Taiwan University, Taipei, Taiwan, 106, Republic of China
}

(Received 25 March 2002; accepted 27 November 2002)

\begin{abstract}
Repeated spike treatment (RST), which was characterized by setting the temperature to ramp up and down repeatedly, was employed to study the stress effect on wafers by annealing the silicon wafers in ambient $\mathrm{N}_{2}$, followed by oxidation in $\mathrm{O}_{2}$. These RST $+\mathrm{O}$ samples had three apparent local thick oxide regions that adjoined the contacts of the three-pin quartz holder, as opposed to the typical $+\mathrm{O}$ samples annealed with a conventional temperature profile before the same oxidation process. It was observed that defects could be created on the silicon surface due to the high thermal stress at contacts during RST, and that the oxidation rate of these damaged zones was greatly increased. $I-V$ data show that for a similar oxide thickness, the leakage currents in RST $+\mathrm{O}$ samples are higher and more scattered than those in typical $+\mathrm{O}$ samples. $C-V$ measurements illustrate that $\mathrm{RST}+\mathrm{O}$ samples have higher interface state densities than typical $+\mathrm{O}$ samples. These enhanced degradation phenomena could be caused by the RST, which resulted in a rough $\mathrm{Si} / \mathrm{SiO}_{2}$ interface and nonuniform oxide thickness. (C) 2003 American Institute of Physics. [DOI: 10.1063/1.1539536]
\end{abstract}

\section{INTRODUCTION}

The continuing downscaling of device dimensions in ultralarge-scale integrated (ULSI) circuits places increasingly challenging demands on the manufacturing tools and technologies for fabricating complex microelectronic chips. Rapid thermal processing (RTP) provides a more promising choice than conventional furnaces due to its short processing time and the consequent reduction in redistribution of the dopant. However, temperature nonuniformity is a major problem in RTP. When a RTP system is used for oxidation or epitaxy, the temperature nonuniformity leads to film nonuniformity, and thermal stress arises from the temperature gradient across the wafer, causing plastic deformation of the wafer. This plastic deformation gives rise to pattern misalignment, device failure, generation of defects in dielectric films, etc. ${ }^{1}$ Much effort has been made to study this problem of thermal stress in RTP. Vandenabeele et al. showed direct experimental evidence that pattern induced temperature nonuniformity could cause plastic deformation. ${ }^{2}$ Lord built up a simple two dimensional model to explore the thermal stress in $\mathrm{RTP}^{3}$ whereas a three dimensional model was employed by Erofeev et al. ${ }^{4}$ and by Kolpakov et al. ${ }^{5}$ Most commercial RTP systems currently achieve their steady state temperature uniformity by shaping the reflector and dynamically controlling the multizone heating sources. However, the thermal stress during the transient state becomes critical. Campbell and Knutson ${ }^{6}$ and Jongste et al. ${ }^{7}$ pointed out that the thermal stress sustained by a wafer was more severe in the transient than in the steady state. Moreover, most past studies have focused on the wafer temperature gradient due to nonuniform heating by lamps, so the effect due to the contact points between the wafer and wafer holder has still not been studied. In this work, a method called repeated spike treatment

${ }^{a)}$ Corresponding author; electronic mail: hwu@cc.ee.ntu.edu.tw
(RST), characterized by setting the temperature to ramp up and down repeatedly, was used to anneal the wafer in inert gas before oxidation. By means of the RST, degradation caused by thermal stress at contact points in RTP was enhanced and clearly demonstrated.

\section{EXPERIMENT}

In this work, 3 in. (100) $p$-type silicon wafers with a resistivity of $1-5 \Omega \mathrm{cm}$ and a thickness of $500 \mu \mathrm{m}$ were used. After cutting them in half and standard RCA cleaning, the typical $+\mathrm{O}$ samples were subjected to the temperature profile shown in Fig. 1 in 20 Torr $\mathrm{N}_{2}$, and then oxidized in 30 Torr $\mathrm{O}_{2}$ at $850^{\circ} \mathrm{C}$ for 10,20 , and $30 \mathrm{~s}$, respectively, in a RTP system. The RST $+\mathrm{O}$ samples were prepared similarly to the typical $+O$ samples, but under the temperature profile, before oxidation, shown in Fig. 2. The oxide thickness was measured with an ellipsometer by setting the refractive index at 1.46 , and the distance between data points was $3 \mathrm{~mm}$. After Al film deposition, metal-oxide-semiconductor (MOS) capacitors $150 \times 150 \mu \mathrm{m}^{2}$ were formed by conventional photolithography. The MOS current-voltage $(I-V)$ curves were measured with an HP 4154 and the capacitance-voltage $(C-V)$ curves with an HP 4286.

\section{RESULTS AND DISCUSSION}

The oxide thickness distributions of the typical $+\mathrm{O}$ and $\mathrm{RST}+\mathrm{O}$ samples, which were oxidized in 30 Torr $\mathrm{O}_{2}$ at $850{ }^{\circ} \mathrm{C}$ for $30 \mathrm{~s}$, are shown in Figs. 1 and 2, respectively. A schematic diagram of a half wafer on the holder is also shown for better understanding, and the position of wafer orientation flat $(\mathrm{OF})$ is clearly indicated. The uniformity of the oxide thickness of the typical $+\mathrm{O}$ sample seems fine. It had previously been reported that the quartz holder acted as a heat sink during oxidation, causing a thinner oxide near the contact point than elsewhere on the wafer in the thick oxide 


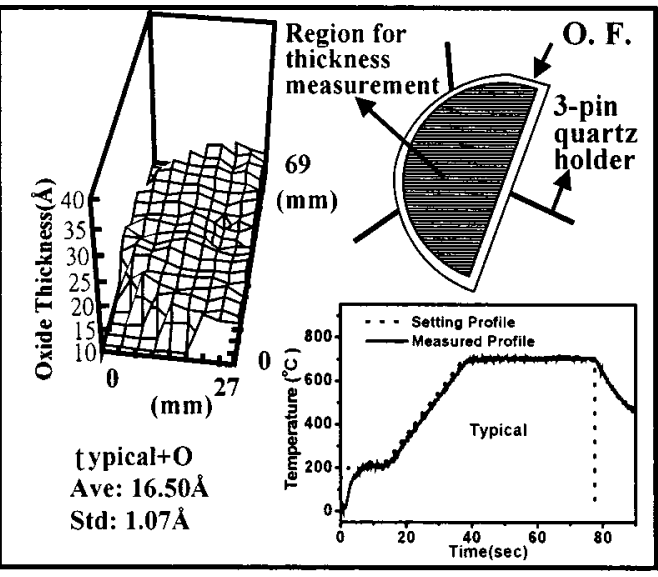

FIG. 1. Oxide thickness distribution on half a 3 in. wafer and the temperature profile used to treat the wafer before oxidation in a typical $+\mathrm{O}$ sample. The schematic diagram shows the relative locations of the wafer and threepin quartz holder during treatment and oxidation.

regime. ${ }^{8,9}$ However, this effect was not observed in the thickness distribution illustrated in Fig. 1, possibly because the oxide obtained was relatively thin. In contrast, the oxide thickness distribution of the RST $+\mathrm{O}$ sample illustrated in Fig. 2 under the same oxidation condition shows clearly different behavior. Here, there are three local thick regions, and each region just adjoined the corresponding contact point with the three-pin quartz holder. The thickness profiles of the $\mathrm{RST}+\mathrm{O}$ samples oxidized at $850{ }^{\circ} \mathrm{C}$ for 10 and $20 \mathrm{~s}$ showed the same tendency. Silicon and quartz have different thermal properties, such as thermal conductivity, specific heat, emissivity, thermal expansion coefficient, etc. Although the heat transfer mechanism between wafer and quartz in direct contact was not clearly specified, the concept of nonuniform heating could be properly applied. The wafer in contact with a three-pin quartz holder during the thermal cycle could be considered a superposition of a floating wafer and three additional heat elements that interact with the wafer as heat sources or sinks. These three heat elements would cause a temperature gradient, and hence thermal stress on the wafer

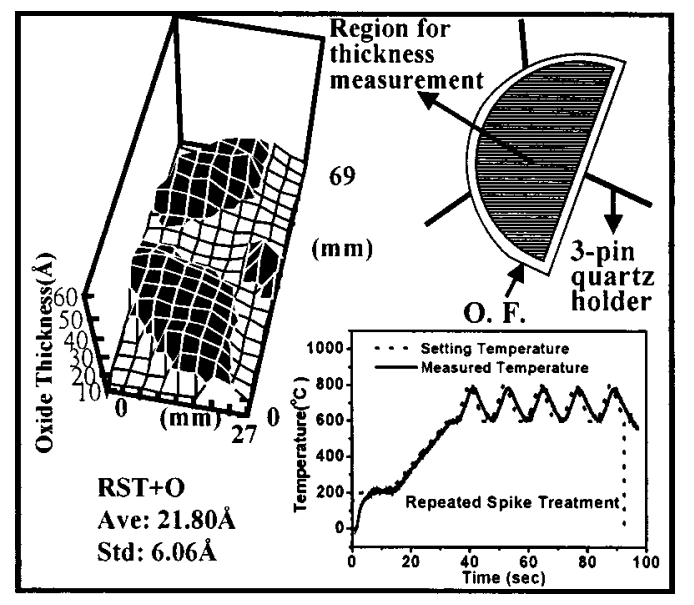

FIG. 2. Oxide thickness distribution on half a 3 in. wafer and the temperature profile used to treat the wafer before oxidation in a RST $+\mathrm{O}$ sample. The schematic diagram shows the relative locations of the wafer and threepin quartz holder during treatment and oxidation.

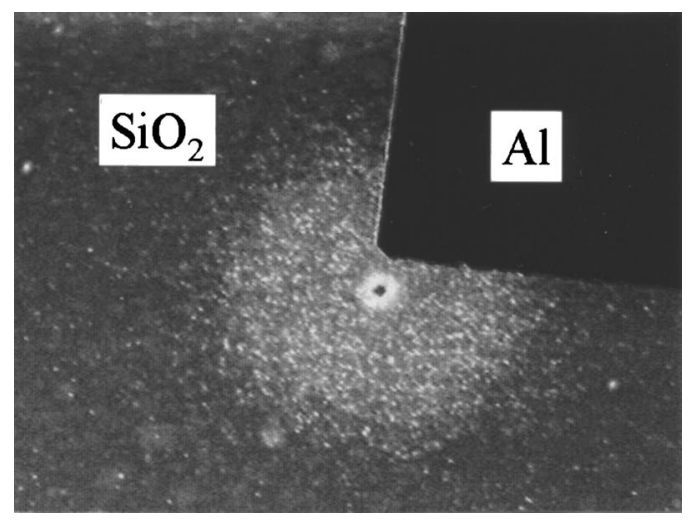

FIG. 3. Photograph of defects on RST $+\mathrm{O}$ samples.

during thermal cycles. If the contact points act as heat sources (sinks) during thermal cycles, it would lead to regions of wafer having relatively high (low) stress and suffer from compressive (tensile) stress. ${ }^{7}$ The RST with repeated temperature ramps up and down enhances the transient effect during a thermal cycle and exacerbates the thermal stress problem caused by contact with the three-pin quartz holder. If the thermal stress exceeds the critical value that the silicon wafer can withstand, defects will be created in the bulk and on the surface. The oxidation rate of silicon would be enhanced where damage had formed previously. ${ }^{10,11}$ The enhanced oxidation rate due to the damaged silicon could be simply expressed as ${ }^{12}$

$$
\frac{B}{A}=R_{1}+K C_{V^{T}},
$$

where $B / A$ is the linear rate constant of oxidation that dominates in the thin oxide regime, $R_{1}$ represents all mechanisms other than the vacancy driven process, $K$ is a proportional constant and $C_{V^{T}}$ is the total vacancy population in silicon. Thus, an oxidation step following the RST process would show the damaged regions on the wafer caused by the RST by the clear appearance of thicker oxide near the contact points. Campbell and Knutson ${ }^{6}$ reported that the magnitude of thermal stress reaches a maximum during temperature ramp up, and decreases during steady state temperature. In addition, the faster the ramp up rate, the higher the magnitude of the thermal stress maximum. Jongste et al. ${ }^{7}$ also noted that during both the ramp up and the ramp down, the inverse radius curvature of wafer, i.e., the stress that the wafer suffered, was higher than that at the steady state temperature. The observation here that the thermal stress problem was more pronounced during temperature ramp up and down was inconsistent with their studies.

Figure 3 is a photograph of defects on the RST $+\mathrm{O}$ sample. Mist-like defects can be seen on the RST $+\mathrm{O}$ samples by the naked eye. Under a microscope the defects appear to be concentric circles. These defects are distributed over the entire surface of all the RST $+\mathrm{O}$ samples, and are especially dense near contact points with the three-pin quartz holder. The distribution and shape of defects are different from those reported in previous studies which illustrated thermal stress induced slip occurring around the wafer pe- 


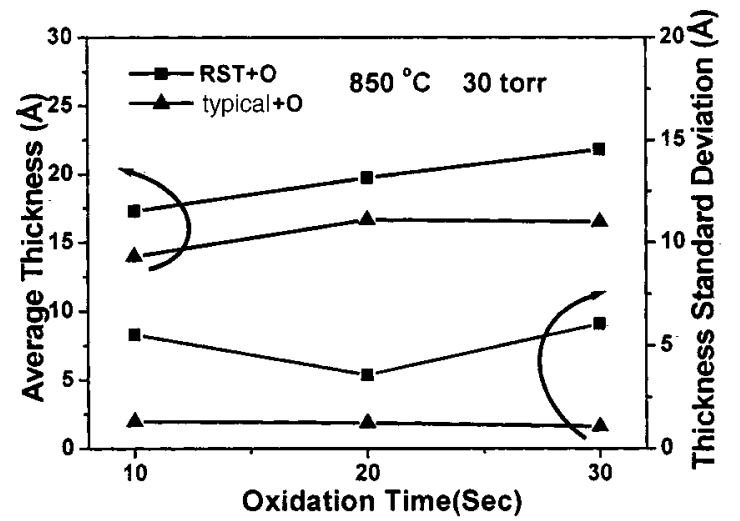

FIG. 4. Average oxide thickness and standard deviation of typical $+\mathrm{O}$ and $\mathrm{RST}+\mathrm{O}$ samples vs the oxidation time.

riphery and were equally spaced at an angle of $22.5^{\circ} \cdot .^{13,14}$ It was assumed that the temperature distribution on a wafer was radius dependent and angle independent in cylindrical coordinates, and thus slip occurred symmetrically around the wafer periphery. ${ }^{13,14}$ However, this analysis is not applicable when the heat transfer caused by the contact points is taken into account.

Figure 4 shows the average and standard deviations in oxide thickness for both $\mathrm{RST}+\mathrm{O}$ and typical $+\mathrm{O}$ samples. It can be observed that under the same oxidation conditions, the RST $+\mathrm{O}$ samples exhibit larger average thickness values and standard deviation than typical $+O$ samples. It is suggested that the RST damages the wafer, especially near contact points with the three-pin quartz holder, and the damaged regions in silicon have a faster oxidation rate.

In order to confirm the oxide qualities of the RST $+\mathrm{O}$ and typical $+\mathrm{O}$ samples, $I-V$ and $C-V$ measurements of MOS devices were performed. Since all RST $+\mathrm{O}$ samples had large variations in oxide thickness, in order to compare the leakage currents of MOS devices with those of the $\mathrm{RST}+\mathrm{O}$ and typical $+\mathrm{O}$ samples, devices with similar oxide thickness were chosen and tested. The 20 MOS devices measured on each sample were located within an area $3 \mathrm{~mm} \times 3$ $\mathrm{mm}$, since $3 \mathrm{~mm}$ was the distance between the thickness data points measured by ellipsometry. Figure 5 illustrates the

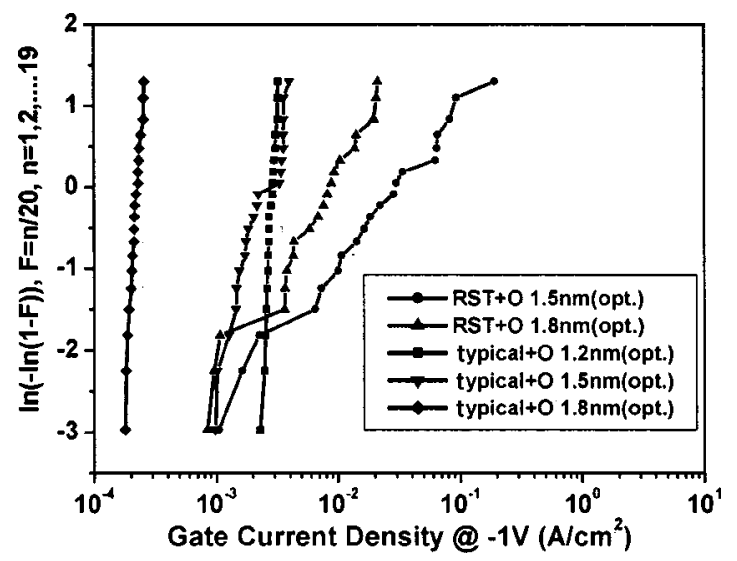

FIG. 5. Weibull plots of tunneling current densities under $-1 \mathrm{~V}$ bias for $\mathrm{RST}+\mathrm{O}$ and typical $+\mathrm{O}$ samples with various oxide thicknesses.

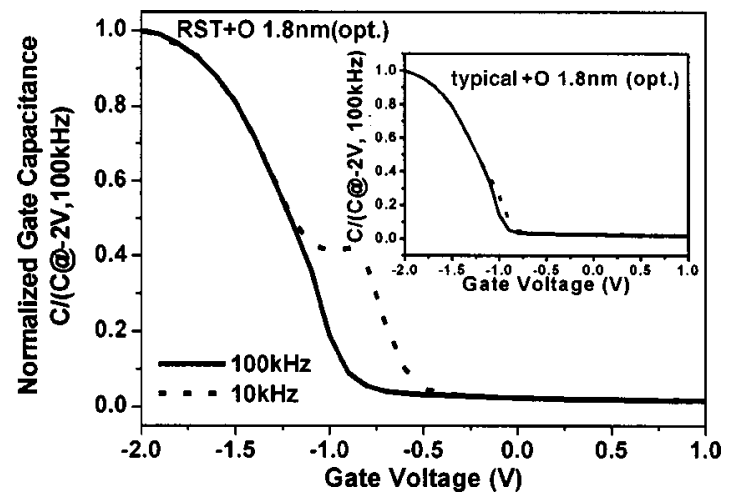

FIG. 6. Normalized $C-V$ curves for $\mathrm{RST}+\mathrm{O}$ and typical $+\mathrm{O}$ samples measured at various frequencies.

Weibull plots of leakage current densities at $-1 \mathrm{~V}$ gate bias for various $\mathrm{RST}+\mathrm{O}$ and typical $+\mathrm{O}$ samples. By comparing samples having similar oxide thicknesses, it can be seen that the distributions of all typical $+\mathrm{O}$ samples seem to be quite normal, but the leakage currents of the 1.5 and $1.8 \mathrm{~nm}$ $\mathrm{RST}+\mathrm{O}$ samples are even higher than those of the thinner $1.2 \mathrm{~nm}$ typical $+\mathrm{O}$ sample, and are widely distributed over about two to three orders of magnitude. The MOS leakage current was very sensitive to oxide thickness and uniformity. ${ }^{15,16}$ Generally, a rougher oxide in a MOS device would cause a higher leakage current. Since the silicon surface of a RST $+\mathrm{O}$ sample is damaged by the RST before oxidation, it is obvious that a rough $\mathrm{Si} / \mathrm{SiO}_{2}$ interface and nonuniform oxide thickness would be obtained after the oxidation step. Normalized $C-V$ curves measured at two frequencies of the $\mathrm{RST}+\mathrm{O}$ and typical $+\mathrm{O}$ samples are shown in Fig. 6. By examining the $C-V$ curves under the depletion region, we can say that the interface state density was higher in the $\mathrm{RST}+\mathrm{O}$ sample compared to in the typical $+\mathrm{O}$ sample having similar oxide thickness. This also supports the inference that the RST $+\mathrm{O}$ samples had a rougher $\mathrm{Si} / \mathrm{SiO}_{2}$ interface than typical $+\mathrm{O}$ ones.

\section{CONCLUSIONS}

Thermal stress induced degradation at contacts of the wafer with its holder during RTP was investigated in this work. With the aid of the RST technique, the transient effect in the RTP was enhanced and the thermal stress caused by contact points was exaggerated by the intentionally applied temperature ramps. The oxide thickness distribution and the reliability of MOS devices were degraded by defects on the silicon wafer caused by thermal stress. This investigation emphasizes the importance of thermal stress caused by contacts during RTP in ULSI technology.

\section{ACKNOWLEDGMENTS}

The authors thank the National Science Council of the Republic of China for supporting this work under Contract No. NSC 90-2212-E-002-225 and Professor I.-Kong Fong of the National Taiwan University for assistance in developing the hardware for the repeated spike function. 
${ }^{1}$ R. P. S. Thakur, N. Chhabra, and A. Ditali, Appl. Phys. Lett. 64, 3428 (1994).

${ }^{2}$ P. Vandenabeele, K. Maex, and R. D. Keersmaecker, Mater. Res. Soc. Symp. Proc. 146, 149 (1989).

${ }^{3}$ H. A. Lord, IEEE Trans. Semicond. Manuf. 1, 105 (1988).

${ }^{4}$ A. F. Erofeev, T. M. Makhviladze, A. V. Panjukhin, O. S. Volchek and O. Adetutu, Proceedings of the 4th International Rapid Thermal Processing Conference, 1996, p. 342,

${ }^{5}$ A. V. Kolpakov, T. M. Makhviladze, A. V. Panjukhin, O. S. Volchek, and A. F. Erofeev, Tech. Dig. - Int. Electron Devices Meet. 541, (1994).

${ }^{6}$ S. A. Campbell and K. L. Knutson, IEEE Trans. Semicond. Manuf. 5, 302 (1992).
${ }^{7}$ J. F. Jongste, T. G. M. Oosterlaken, G. C. J. Bart, G. C. A. M. Jannssen, and S. Radelaar, J. Appl. Phys. 75, 2830 (1994).

${ }^{8}$ K. C. Lee, H. Y. Chang, H. Chang, J. G. Hwu, and T. S. Wung, IEEE Trans. Semicond. Manuf. 12, 340 (1999).

${ }^{9}$ C. C. Hong, Y. R. Yen, J. L. Su, and J. G. Hwu, IEEE Trans. Semicond. Manuf. 1, 102 (2002).

${ }^{10}$ S. M. Hu, Appl. Phys. Lett. 42, 872 (1983).

${ }^{11}$ S. Dunham, J. Appl. Phys. 71, 685 (1992).

${ }^{12}$ C. P. Ho and J. D. Plummer, J. Electrochem. Soc. 126, 1516 (1979).

${ }^{13}$ M. M. Moslehi, IEEE Trans. Semicond. Manuf. 2, 130 (1989).

${ }^{14}$ G. Bentini, L. Correra, and C. Donolato, J. Appl. Phys. 56, 2922 (1984).

${ }^{15}$ B. Majkusiak and A. Strojwas, J. Appl. Phys. 74, 5638 (1993).

${ }^{16}$ Z. A. Weinberg, J. Appl. Phys. 53, 5052 (1982). 